\title{
A comparison of the delivery of inhaled drugs by jet nebulizer and vibrating mesh nebulizer using dual-source dual-energy computed tomography in rabbits: a preliminary in vivo study
}

\author{
Qixing Wang, Yuhao Liu, Zhengfang Zhu, Jian Hu, Li Li, Sheng Wang \\ Department of Critical Care Medicine, Shanghai Tenth People's Hospital, Tongji University School of Medicine, Shanghai, China \\ Contributions: (I) Conception and design: Q Wang, S Wang; (II) Administrative support: S Wang; (III) Provision of study materials or patients: Y \\ Liu, Z Zhu; (IV) Collection and assembly of data: Q Wang, J Hu, L Li; (V) Data analysis and interpretation: Q Wang, J Hu, L Li; (VI) Manuscript \\ writing: All authors; (VII) Final approval of manuscript: All authors. \\ Correspondence to: Sheng Wang. Department of Critical Care Medicine, Shanghai Tenth People's Hospital, Tongji University School of Medicine, \\ Shanghai, China. Email: shengwang26@163.com.
}

\begin{abstract}
Background: Radionuclide imaging is the primary method of visually evaluating drug deposition in the lungs. Here, we used a new imaging technique, dual-source dual-energy computed tomography (DSDECT), to compare the ability of two nebulizers commonly used in clinical practice to deposit drugs in the lungs.

Methods: Male New Zealand White rabbits were randomly divided into a vibrating mesh nebulizer group and a small volume jet nebulizer group. First, two rabbits received methylene blue-iohexol-normal saline by inhalation to confirm that DSDECT was a feasible method of assessing aerosol inhalation. Then, 10 rabbits were subjected to DSDECT scan before and after receiving a iohexol-normal saline mixture by inhalation. All images were transferred and reconstructed on a workstation computer equipped with dedicated software. Results: DSDECT revealed the same iodine distribution as tracheobronchial methylene blue staining on dissection. Significant differences between the groups in average CT value and iodine concentration were noted in the right upper lobe $(\mathrm{P}<0.05)$, but no other lobes showed statistically significant differences. The regional distribution of drugs in different lobes of the lung varied by nebulizer.

Conclusions: DSDECT is a useful method of evaluating drug deposition in the lungs, and vibrating mesh nebulizers deposit more drugs than small volume jet nebulizers.
\end{abstract}

Keywords: Drug aerosol therapy; lung deposition; dual-source dual-energy computed tomography; nebulizer

Submitted Feb 14, 2020. Accepted for publication Jul 24, 2020.

doi: $10.21037 / \mathrm{atm}-20-1584$

View this article at: http://dx.doi.org/10.21037/atm-20-1584

\section{Introduction}

Aerosol inhalation is a well-known method of delivering drugs to the lungs of a patient receiving mechanical ventilation. Delivering drugs via the pulmonary route has several advantages, including increased local concentration of the drug in the lungs, improved lung receptor occupancy, increased absorption due to the large surface area, reduced local and systemic drug delivery, and reduced systemic adverse reactions $(1,2)$.

Systems for pulmonary drug delivery for mechanically ventilated patients include metered-dose inhalers, small volume jet nebulizers (SVJNs), and vibrating mesh nebulizers (VMNs). The latter two liquid nebulization systems are more commonly used in clinical practice. Although studies have shown that SVJNs and VMNs differ in terms of price, deposition rate, particle size produced, and aerosol drug output, VMNs outperform SVNs in the total amount of drug deposited in the lungs. Yet, jet nebulizers remain the most frequently used nebulizers for administering drugs to patients on mechanical ventilation $(3,4)$.

The target area for inhalation therapy varies by disease. Whereas asthma patients need drugs delivered to the 


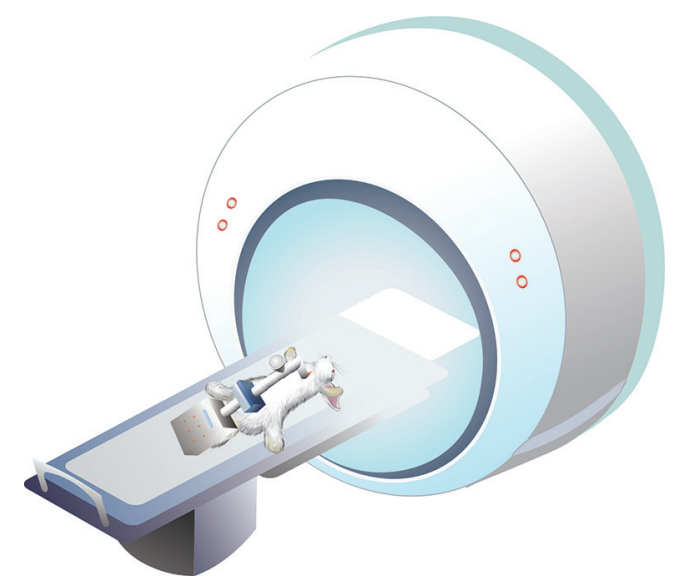

Figure 1 In vivo model of the delivery of inhaled drugs by jet nebulizer and vibrating mesh nebulizer via dual-source dual-energy computed tomography.

bronchi and bronchial airway (5), pneumonia patients may benefit from the delivery of drugs to the alveolar region. Hence, not only the total pulmonary drug dose but the regional distribution or aerosol deposition distribution is a key factor in the clinical success of inhalation therapy.

Lung deposition data play a vital role in assessing the efficiency of systems that deliver inhaled drugs. Radionuclide imaging is the primary method of visually evaluating drug deposition in the human airway and plays a role in the development of new inhaled medications and delivery devices (6). However, it has the disadvantage of exposing subjects and operators to health risks or even cancer due to the use of ionizing radiation (7-9). Low resolution and time constraints due to the decay of the radionuclide also limit its use as the gold standard for measuring lung deposition (10).

A noninvasive, next-generation imaging technique, dualsource dual-energy computed tomography (DSDECT), is increasingly used in clinical diagnosis. The advantages of DSDECT are that it permits free breathing without breathhold, produces powerful images, and only needs a very small dose of contrast media. Especially for regional ventilation, DSDECT's spatial resolution is much higher than that of radionuclide scanning imaging equipment. It also offers the ability to demine the iodine content of a lesion or region.

No studies to date have reported on the efficacy of DSDECT for assessing drug deposition in the lungs. Here, we used an animal model of mechanical ventilation to compare the ability of two nebulizers to deposit inhaled drugs in the lungs. We used conventional contrast agents and relatively safe (low-radiation), noninvasive, highresolution dual-energy CT. We present the following article in accordance with the ARRIVE reporting checklist (available at http://dx.doi.org/10.21037/atm-20-1584).

\section{Methods}

\section{Ethics statement}

Animal care and all animal experiments were approved by the Animal Ethics Committee of Shanghai Tenth People's Hospital, Tongji University School of Medicine, and adhered to local animal care guidelines.

\section{Animals}

Twelve male New Zealand White rabbits (2.6-3.4 kg; Shanghai Jiagan Biotechnology Co., Ltd, China) were used. They were housed in individual cages in a room with constant humidity and temperature under a $12 \mathrm{~h}$ light-dark cycle and fed a standard rabbit diet and ad libitum.

\section{DSDECT scanning}

Rabbits were randomly divided into two groups (VMN and SVJN), with six rabbits in each group. They were anesthetized via intraperitoneal injection of $5 \%$ chloral hydrate and a tracheostomy was performed. A tracheostomy tube of $4.0 \mathrm{~mm}$ inside diameter was inserted into the trachea. We used an infant-use ventilator and circuits in conjunction with the experimental nebulizer placed in the inspiratory limb of the circuit as recommended (11). The experimental settings are shown in Figure 1. To avoid the interference of iodine in the exhaled breath with the imaging, we used a new breathing system filter (Ultipor 100; Pall Corporation, Ann Arbor, MI, USA) for each rabbit test. All lung CT images were obtained via a thirdgeneration dual-source CT scanner (SOMATOM Force; Siemens Healthcare, Forchheim, Germany). Baseline CT of the chest was performed before aerosol therapy.

We first wanted to confirm that DSDECT was a feasible method of assessing aerosol inhalation. Therefore, two rabbits received $2 \mathrm{~mL}$ methylene blue-iohexol-normal saline (1:1:5) by inhalation before DSDECT scan. After scanning, the rabbits were euthanized and their lungs were rapidly removed and dissected longitudinally from the center of the bronchus along the coronal plane.

For the VMN group, nebulization continued until no 
further mist was visible. For the SVJN group, nebulization continued until the sputter sound with inconsistent nebulization. After that, the rabbits received a non-ionic, low osmolar contrast medium containing $2 \mathrm{~mL}$ iohexolnormal saline (1:6) by inhalation. DSDECT of the lungs was performed immediately after the iohexol aerosol inhalation. At the same time, the nebulization time was recorded.

\section{Image reconstruction and analysis}

All dual-energy images were transferred and reconstructed on a workstation equipped with dedicated software (Syngo. via version VB10B; Siemens Healthcare). Three images were taken: a $70 \mathrm{kV}$ image, an $\mathrm{Sn} 150 \mathrm{kV}$ image, and a weighted image with $60 \%$ density from the $70 \mathrm{kV}$ image and $40 \%$ density from the $\mathrm{Sn} 150 \mathrm{kV}$ image. Ventilation images were obtained based on the material decomposition theory. The material parameters were as follows: $-1,000 \mathrm{HU}$ for air at $70 \mathrm{kVp}, 60 \mathrm{HU}$ for soft tissue at $70 \mathrm{kVp},-1,000 \mathrm{HU}$ for air at $\mathrm{Sn} 150 \mathrm{kVp}$, and $54 \mathrm{HU}$ for soft tissue at $\mathrm{Sn} 150 \mathrm{kVp}$. The relative contrast material enhancement was 2 , with a minimum value of $-960 \mathrm{HU}$ and a maximum value of $-400 \mathrm{HU}$; the range was 4 .

All images were independently and blindly evaluated by two chest radiologists with more than 10 years of experience in interpreting chest CT scans. Morphological changes and ventilation images were assessed for each rabbit for each of the five lobes of the lung: right upper, right middle, right lower, left upper, and left lower.

The standard approach to evaluating local concentrations of a radiolabeled substance is to measure the average activity per unit volume within defined regions of interest (ROIs). ROIs are binary masks that group voxels corresponding to specific anatomical regions. An experienced radiologist placed circular ROIs $0.2 \mathrm{~cm}^{2}$ in the lobes. The average $\mathrm{CT}$ value (HU) and average iodine density $\left(\mu \mathrm{g} / \mathrm{cm}^{3}\right)$ were captured in the ROI. The overlay value, which is a DSDECT measure of iodine concentration on iodine maps developed by Siemens, was measured four times to quantify the iodine content in the lobes. The results were averaged as a final measure of overlay.

\section{Statistical analyses}

Statistical analyses were performed with SPSS (IBM, Armonk, NY, USA) and Prism 8 (GraphPad Software, San Diego, CA, USA). DSDECT images were outputted from the workstation and read using RadiAnt DICOM Viewer
5.5.1 (RadiAnt Dicom Viewer, Medixant, Poznan, Poland). Student's $t$ tests were used for between-groups comparisons, and one-way analyses of variance were used for multigroup comparisons. $\mathrm{P}<0.05$ was considered statistically significant. Violin plots were generated with Prism.

\section{Results}

Two rabbits were used to clarify the relationship between methylene blue staining and dual-source dual-energy iodine imaging. One rabbit in the SVJN group was excluded because it died during the study. Thus, nine rabbits were included in the final data analyses: four in the SVJN group and five in the $\mathrm{VMN}$ group.

\section{The relationship between methylene blue staining and dual-energy iodine imaging}

DSDECT scan after the inhalation of methylene blueiohexol-normal saline $(1: 1: 5)$ revealed the same iodine distribution as tracheobronchial methylene blue staining on dissection, with deeper staining and a greater concentration of iodine in the right upper lobe, as shown in Figure 2.

\section{Average change in $C T$ value and iodine density between the groups}

Quantitative analyses showed that the baseline average CT value and average iodine density were similar between the two groups, with no statistically significant differences (Tables 1,2). After inhalation of the iohexol mixture, the average CT value decreased slightly and iodine density increased in the right middle, right lower, left upper, and left lower lobes, but no statistically significant differences were observed. However, significant differences between the two groups in average $\mathrm{CT}$ value and iodine density were noted in the right upper lobe $(\mathrm{P}=0.024)$, as shown in Tables 3,4 . The mean change in average CT value in the right upper lobe was $-42.694 \pm 89.986 \mathrm{HU}$ in the SVJN group and $124.120 \pm 83.633 \mathrm{HU}$ in the VMN group $(\mathrm{P}=0.024$; Table 3$)$. The mean change in average iodine density in the right upper lobe was $0.400 \pm 0.293 \mu \mathrm{g} / \mathrm{cm}^{3}$ in the SVJN group and $1.575 \pm 0.533 \mu \mathrm{g} / \mathrm{cm}^{3}$ in the VMN group $(\mathrm{P}=0.005$; Table 4$)$.

\section{DSDECT deposition data following iobexol-based aerosol therapy}

On iodine maps of DSDECT, the brighter the color, the 

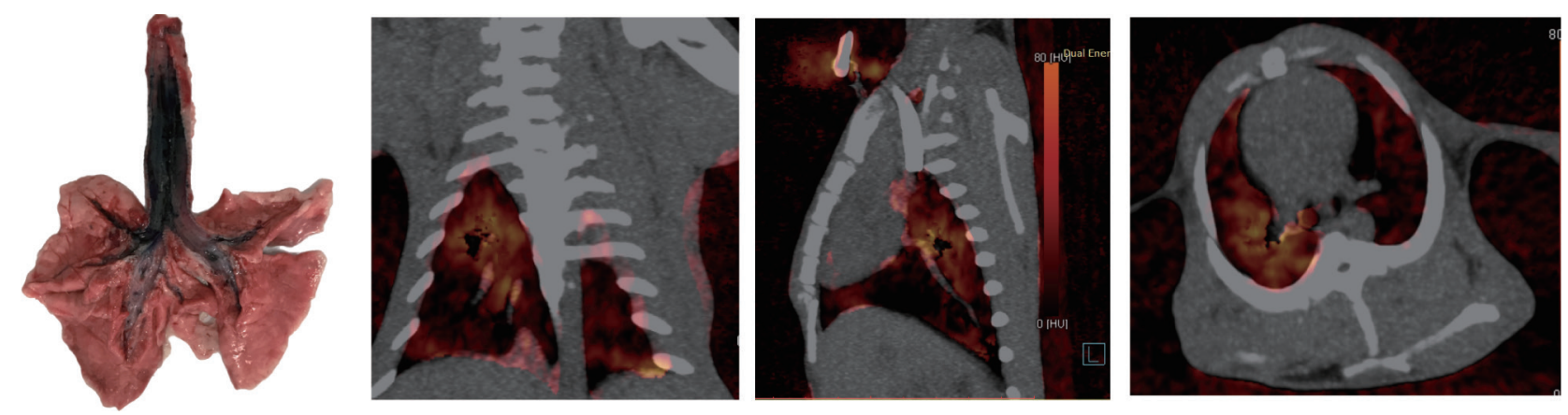

Figure 2 Methylene blue staining and dual-energy iodine imaging in rabbits illustrating the good relationship between the two methods. Iodine is indicated in red. The iodine overlay image is superimposed on a gray-scale mixed image.

Table 1 Baseline computed tomography values in the two groups

\begin{tabular}{lccc}
\hline Lobe & VMN $(n=5)$ & $\operatorname{SVJN}(n=4)$ & $P$ \\
\hline RUL & $-675.405 \pm 66.606$ & $-585.494 \pm 125.650$ & 0.207 \\
RML & $-720.465 \pm 53.165$ & $-645.806 \pm 66.497$ & 0.095 \\
RLL & $-565.325 \pm 57.206$ & $-533.038 \pm 27.775$ & 0.310 \\
LUL & $-663.305 \pm 70.499$ & $-597.100 \pm 74.877$ & 0.215 \\
LLL & $-570.360 \pm 76.060$ & $-524.031 \pm 49.556$ & 0.330
\end{tabular}

Data are shown as mean $\pm \mathrm{SD}$. VMN, vibrating mesh nebulizer; SVJN, small volume jet nebulizer; RUL, right upper lobe; RML, right middle lobe; RLL, right lower lobe; LUL, left upper lobe; LLL, left lower lobe.

Table 2 Baseline iodine concentrations in the two groups

\begin{tabular}{lccc}
\hline Lobe & VMN $(n=5)$ & $\operatorname{SVJN}(n=4)$ & $P$ \\
\hline RUL & $1.930 \pm 0.703$ & $1.481 \pm 0.394$ & 0.295 \\
RML & $1.730 \pm 0.390$ & $1.575 \pm 0.206$ & 0.499 \\
RLL & $2.415 \pm 0.702$ & $1.600 \pm 0.235$ & 0.059 \\
LUL & $2.375 \pm 0.747$ & $1.606 \pm 0.815$ & 0.184 \\
LLL & $2.165 \pm 0.645$ & $1.669 \pm 0.642$ & 0.288 \\
\hline
\end{tabular}

Data are shown as mean $\pm \mathrm{SD}$. VMN, vibrating mesh nebulizer; SVJN, small volume jet nebulizer; RUL, right upper lobe; RML, right middle lobe; RLL, right lower lobe; LUL, left upper lobe; LLL, left lower lobe.

higher the iodine concentration. The iodine concentration was high in the VMN group (bottom row of Figure 3) in overall views of the coronal plane (left) and axial plane (middle) and three-dimensional reconstruction images, as shown in Figure 3. The DSDECT images also have high resolution.
Table 3 Computed tomography values after iohexol inhalation in the two groups

\begin{tabular}{lccc}
\hline Lobe & VMN $(n=5)$ & $\operatorname{SVJN}(n=4)$ & $P$ \\
\hline RUL & $124.120 \pm 83.633$ & $-42.694 \pm 89.986$ & $0.024^{*}$ \\
RML & $47.565 \pm 93.917$ & $-36.106 \pm 23.326$ & 0.130 \\
RLL & $30.510 \pm 46.762$ & $-29.263 \pm 32.777$ & 0.068 \\
LUL & $64.705 \pm 39.606$ & $21.106 \pm 101.196$ & 0.401 \\
LLL & $56.525 \pm 88.341$ & $-14.975 \pm 54.682$ & 0.202 \\
\hline
\end{tabular}

Data are shown as mean $\pm \mathrm{SD}$. *, $\mathrm{P}<0.05$. VMN, vibrating mesh nebulizer; SVJN, small volume jet nebulizer; RUL, right upper lobe; RML, right middle lobe; RLL, right lower lobe; LUL, left upper lobe; LLL, left lower lobe.

Table 4 Iodine concentrations after iohexol inhalation in the two groups

\begin{tabular}{lccc}
\hline Lobe & VMN $(n=5)$ & $\operatorname{SVJN}(n=4)$ & $P$ \\
\hline RUL & $1.575 \pm 0.533$ & $0.400 \pm 0.293$ & $0.005^{\star}$ \\
RML & $0.775 \pm 93.917$ & $0.388 \pm 0.403$ & 0.183 \\
RLL & $1.620 \pm 1.408$ & $0.356 \pm 0.315$ & 0.116 \\
LUL & $0.835 \pm 0.573$ & $0.588 \pm 0.632$ & 0.557 \\
LLL & $1.905 \pm 1.386$ & $0.338 \pm 0.714$ & 0.070 \\
\hline
\end{tabular}

Data are shown as mean $\pm \mathrm{SD}$. ${ }^{*}, \mathrm{P}<0.05$. VMN, vibrating mesh nebulizer; SVJN, small volume jet nebulizer; RUL, right upper lobe; RML, right middle lobe; RLL, right lower lobe; LUL, left upper lobe; LLL, left lower lobe.

\section{Regional drug distribution in different lobes}

Finally, we analyzed the change in average CT value and iodine density in different lobes of the lungs after iohexol inhalation in each group (Figures 4,5). In the 


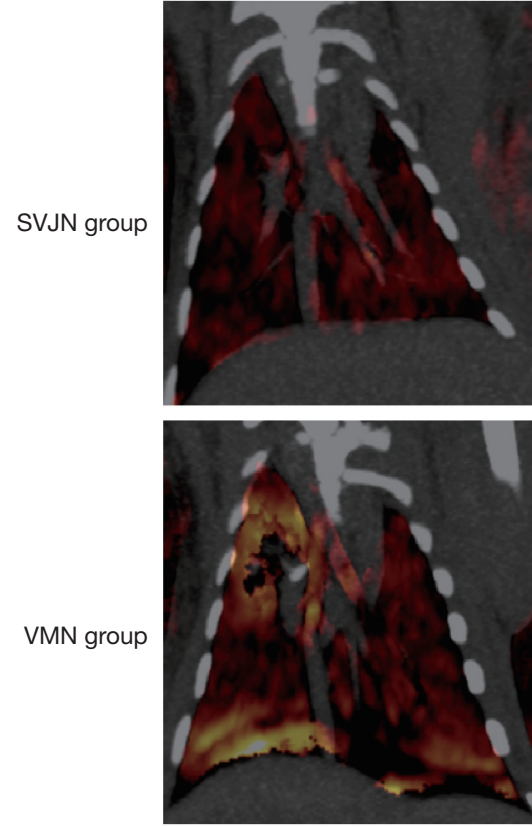

Coronal plane
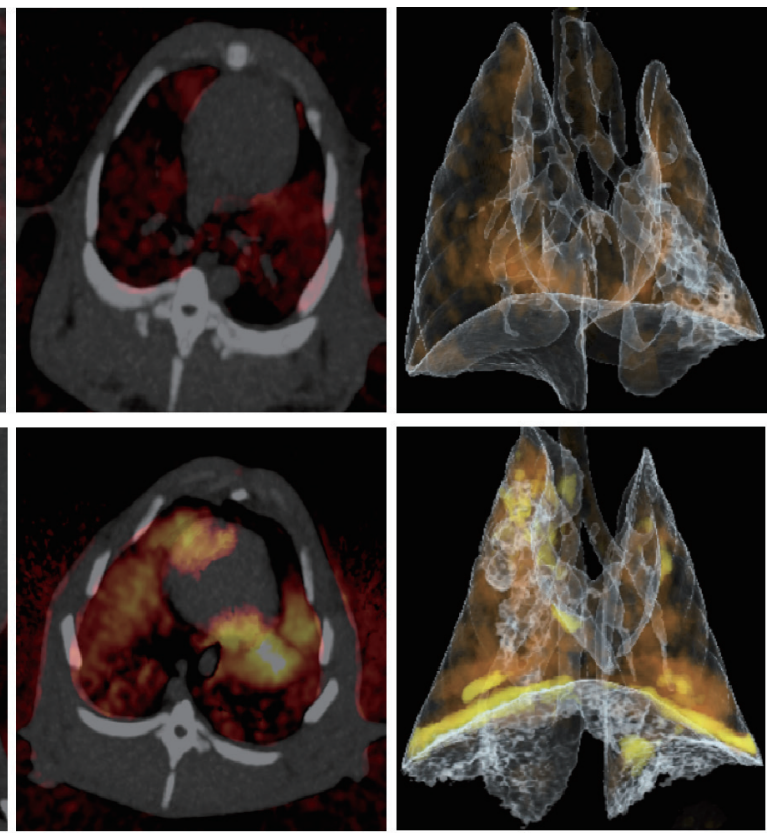

Axial plane

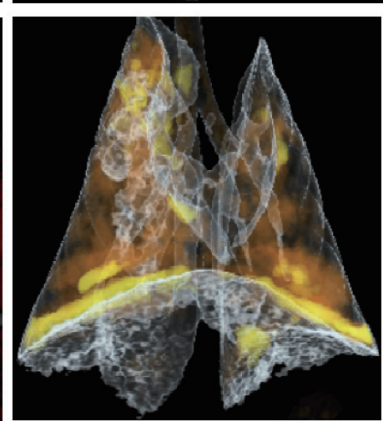

$3 \mathrm{D}$ reconstruction

Figure 3 Dual-energy computed tomography ventilation images illustrating aerosol deposition distribution by small volume jet nebulizers (upper row) and vibrating mesh nebulizers (bottom row) in the lungs of rabbits after iohexol-based aerosol therapy.
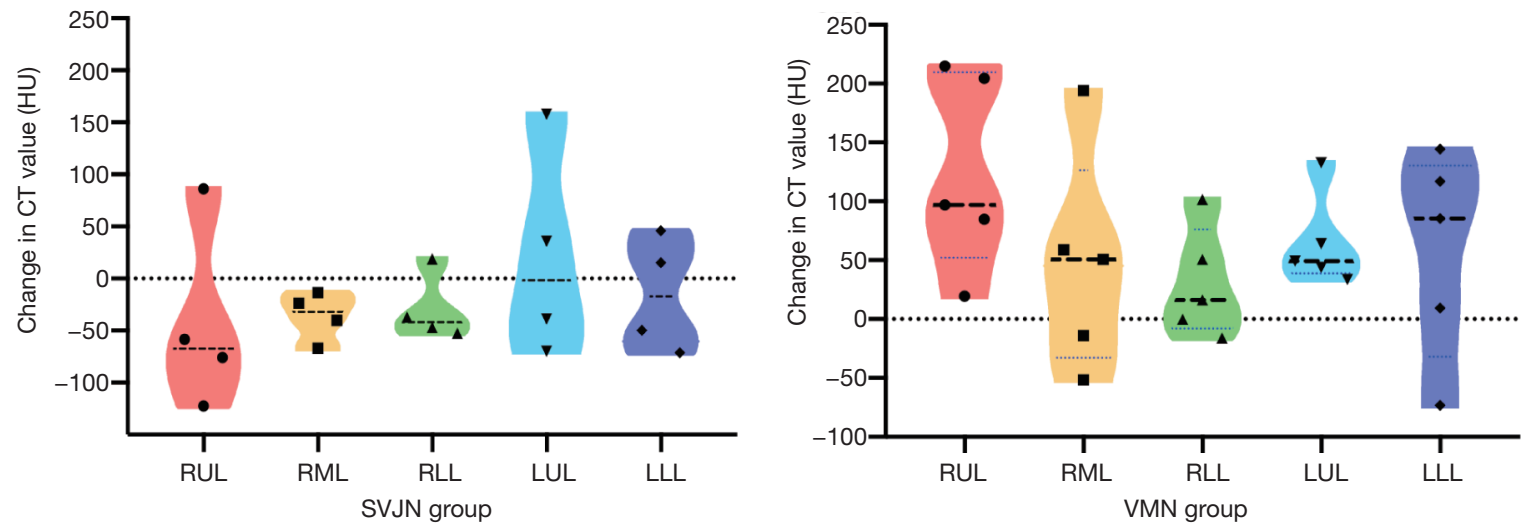

Figure 4 Change in computed tomography values in the lung after iohexol inhalation. SVJN, small volume jet nebulizer; VMN, vibrating mesh nebulizer; RUL, right upper lobe; RML, right middle lobe; RLL, right lower lobe; LUL, left upper lobe; LLL, left lower lobe.

SVJN group, iodine was distributed relatively uniformly in the different lobes. However, in the VMN group, the distribution was uneven: the right upper lobe had a higher iodine concentration than the other lobes. There were no statistically significant differences in the change in average CT value $(\mathrm{P}=0.589)$ or average iodine density $(\mathrm{P}=0.141)$ in the VMN group. The change in both average CT value and iodine density was lower in the SVJN group than in the VMN group.

\section{Discussion}

Here, we used DSDECT to compare the deposition of two nebulizers in a rabbit model of mechanical ventilation. DSDECT uses two independent detector tube systems, records the difference in attenuation of different substances at different levels of energy X-ray, and uses a threesubstance separation algorithm to quantitatively differentiate and quantify materials (12). This technique, which avoids 

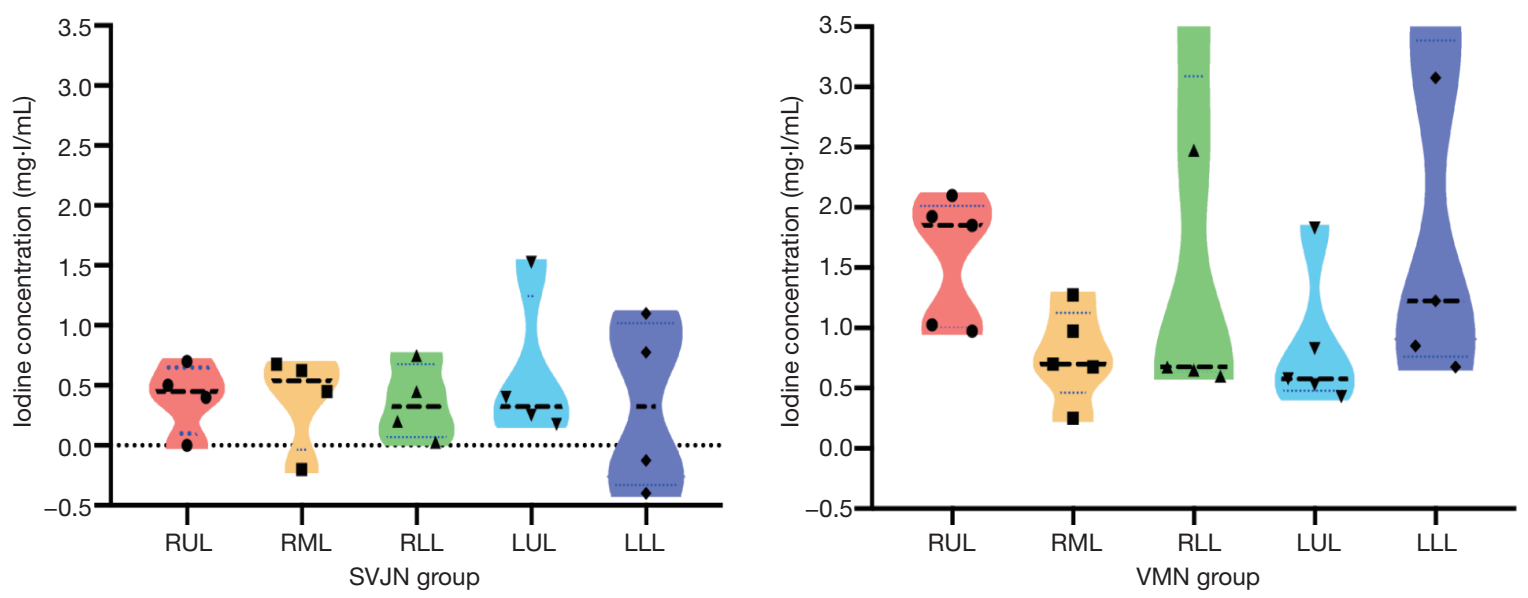

Figure 5 Change in iodine concentrations in the lung after iohexol inhalation. SVJN, small volume jet nebulizer; VMN, vibrating mesh nebulizer; RUL, right upper lobe; RML, right middle lobe; RLL, right lower lobe; LUL, left upper lobe; LLL, left lower lobe.

motion artifacts associated with breathing, is particularly advantageous given the complex three-dimensional structure of the lung. This modality could potentially produce finer resolution images than are available through CT scans and spare patients the significant radiation burden associated with CT. In this study, we used the latest CT scanner, the third-generation dual-source CT SOMATOM Force scanner from Siemens.

There are two major findings from this study. First, the average CT value decreased significantly and the average iodine density increased significantly in the right upper lobe of mechanically ventilated rabbits in the VMN group. This means that more inhaled drugs were deposited in the lungs when a VMN was used. Similar results were obtained in previous studies (13-15). One of the main reasons for this finding is that VMNs have a lower residual volume (the amount of drug that remains in the nebulizer at the end of nebulization) after nebulization and a faster output rate of nebulization than standard SJVNs due to different working principles $(3,4,13)$. Therefore, clinicians should change the dose of the inhaled drug for different nebulizers to avoid overdosing or underdosing. For the same dose of nebulization therapy, more drug was deposited in the lungs by VMN; this is similar to the results of previous radionuclide imaging studies. The atomizing efficiency of VMNs is higher than that of SVJNs.

Second, the distribution of the drug to the lobes of the lung varied by nebulizer under mechanical ventilation. VMNs deposited more drugs in the right upper lobe. This may be because the right main bronchus is relatively straight, and the inhaled aerosol hits the wall and deposits (16).
Or it could be related to the fact that bronchial deposition of drugs depends on the particle diameter: the smaller the particle, the more easily it reaches the periphery of the lung (17). VMNs produce particles ideal for high lung deposition, whereas SVJNs produce smaller particles that are more difficult to deposit. Put another way, SVJNs produce more uniform distribution of inhaled drugs but require an increase in dosage.

The main airway is the largest site of drug deposition. In this study, the research target was invasive mechanical ventilation, both tracheal intubation and tracheotomy. The type of tracheal tube has a minimal, nonsignificant effect on aerosol delivery (18). This study involved tracheotomy in rabbits; deposition in conducting airways, the pulmonary acinus, and the upper airway was not considered. The main reason for not including the main airway in this study is that it is mainly occupied by the artificial airway after endotracheal intubation or tracheotomy, and a large amount of drug adheres to the wall of the artificial airway.

Despite the valuable results from the present study, several limitations should be addressed in future research. Proper contrast agents and doses need further validation in large-scale animal models before being tested in human trials. Also, researchers must determine the proper method for increasing the distribution of inhaled medications in the lungs to improve dose uniformity.

\section{Conclusions}

DSDECT is a useful new technique in the field of clinical CT imaging. The ability of dual-energy and multi-energy 
CT to differentiate materials of different effective atomic numbers and reconstruct them in three dimensions has several new clinically relevant applications. DSDECT that combines imaging agents may provide a new means of evaluating the use of different types of nebulizers in inhalation therapy, but more prospective research is required to provide a basis for clinical applications. This method appears promising for describing regional lung deposition from two commonly used nebulizers during mechanical ventilation in the in vivo model of rabbits.

\section{Acknowledgments}

Funding: This work was supported by the Shanghai Health and Family Planning Commission [grant number 20174Y0148] and National Natural Science Foundation of China [grant number 81601682].

\section{Footnote}

Reporting Checklist: The authors have completed the ARRIVE reporting checklist. Available at http://dx.doi. org/10.21037/atm-20-1584

Data Sharing Statement: Available at http://dx.doi. org/10.21037/atm-20-1584

Peer Review File: Available at http://dx.doi.org/10.21037/ atm-20-1584

Conflicts of Interest: All authors have completed the ICMJE uniform disclosure form (available at http://dx.doi. org/10.21037/atm-20-1584). The authors have no conflicts of interest to declare.

Ethical Statement: The authors are accountable for all aspects of the work in ensuring that questions related to the accuracy or integrity of any part of the work are appropriately investigated and resolved. Animal care and all animal experiments were approved by the Animal Ethics Committee of Shanghai Tenth People's Hospital, Tongji University School of Medicine, and adhered to local animal care guidelines.

Open Access Statement: This is an Open Access article distributed in accordance with the Creative Commons Attribution-NonCommercial-NoDerivs 4.0 International License (CC BY-NC-ND 4.0), which permits the non- commercial replication and distribution of the article with the strict proviso that no changes or edits are made and the original work is properly cited (including links to both the formal publication through the relevant DOI and the license). See: https://creativecommons.org/licenses/by-nc-nd/4.0/.

\section{References}

1. Pison $\mathrm{U}$, Welte T, Giersig M, et al. Nanomedicine for respiratory diseases. Eur J Pharmacol 2006;533:341-50.

2. Mansour HM, Rhee YS, Wu X. Nanomedicine in pulmonary delivery. Int J Nanomedicine 2009;4:299-319.

3. Ehrmann S, Roche-Campo F, Bodet-Contentin L, et al. Aerosol therapy in intensive and intermediate care units: prospective observation of 2808 critically ill patients. Intensive Care Med 2016;42:192-201.

4. Ari A, Fink JB, Dhand R. Inhalation therapy in patients receiving mechanical ventilation: an update. J Aerosol Med Pulm Drug Deliv 2012;25:319-32.

5. Lahzami S, King GG. Targeting small airways in asthma: the new challenge of inhaled corticosteroid treatment. Eur Respir J 2008;31:1145-47.

6. Newman S, Fleming J. Challenges in assessing regional distribution of inhaled drug in the human lungs. Expert Opin Drug Deliv 2011;8:841-55.

7. Fazel R, Shaw LJ. Radiation exposure from radionuclide myocardial perfusion imaging: concerns and solutions. J Nucl Cardiol 2011;18:562-5.

8. Mezrich RS. Cancer risk from multiple imaging tests. JAMA 2011;305:887; author reply 888.

9. Brenner DJ. What we know and what we don't know about cancer risks associated with radiation doses from radiological imaging. Br J Radiol 2014;87:20130629.

10. Chrystyn H. Methods to determine lung distribution of inhaled drugs - could gamma scintigraphy be the gold standard? Br J Clin Pharmacol 2000;49:525-8.

11. Rello J, Sole-Lleonart C, Rouby JJ, et al. Use of nebulized antimicrobials for the treatment of respiratory infections in invasively mechanically ventilated adults: a position paper from the European Society of Clinical Microbiology and Infectious Diseases. Clin Microbiol Infect 2017;23:629-39.

12. McCollough CH, Leng S, Yu L, et al. Dual- and multienergy CT: principles, technical approaches, and clinical applications. Radiology 2015;276:637-53.

13. Dubus JC, Vecellio L, De Monte M, et al. Aerosol deposition in neonatal ventilation. Pediatr Res 2005;58:10-4.

14. Chang KH, Moon SH, Oh JY, et al. Comparison of 
salbutamol delivery efficiency for jet versus mesh nebulizer using mice. Pharmaceutics 2019;11:192.

15. Liu CY, Ko HK, Fink JB, et al. Size distribution of colistin delivery by different type nebulizers and concentrations during mechanical ventilation. Pharmaceutics 2019;11:459.

16. Alzahrany M, Van Rhein T, Banerjee A, et al. Fluid flow and particle transport in mechanically ventilated airways. Part II: Particle transport. Med Biol Eng Comput 2016;54:1097-109.

Cite this article as: Wang Q, Liu Y, Zhu Z, Hu J, Li L, Wang S. A comparison of the delivery of inhaled drugs by jet nebulizer and vibrating mesh nebulizer using dual-source dual-energy computed tomography in rabbits: a preliminary in vivo study. Ann Transl Med 2020;8(17):1072. doi: 10.21037/atm-20-1584
17. Usmani OS, Biddiscombe MF, Barnes PJ. Regional lung deposition and bronchodilator response as a function of beta2-agonist particle size. Am J Respir Crit Care Med 2005;172:1497-504.

18. Dhanani JA, Cohen J, Parker SL, et al. A research pathway for the study of the delivery and disposition of nebulised antibiotics: an incremental approach from in vitro to large animal models. Intensive Care Med Exp 2018;6:17. 